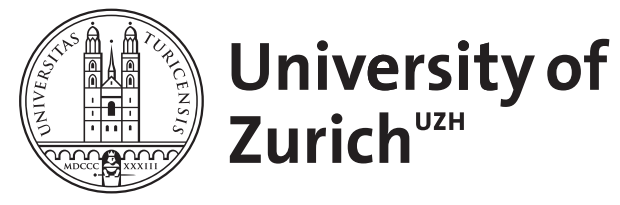

\title{
Wolfgang Pauli and modern physics
}

Straumann, N

\begin{abstract}
In this written version of a pre-dinner-speech at the workshop "The Nature of Gravity" at ISSI I illustrate Pauli's science primarily with material that has not formally been published by him, but was communicated in detailed letters to eminent colleagues and friends
\end{abstract}

DOI: https://doi.org/10.1007/s11214-009-9486-9

Posted at the Zurich Open Repository and Archive, University of Zurich ZORA URL: https://doi.org/10.5167/uzh-156013

Journal Article

Published Version

Originally published at:

Straumann, N (2009). Wolfgang Pauli and modern physics. Space Science Reviews, 148(1-4):25-36.

DOI: https://doi.org/10.1007/s11214-009-9486-9 


\title{
Wolfgang Pauli and Modern Physics
}

\author{
N. Straumann
}

Received: 13 November 2008 / Accepted: 31 December 2009 / Published online: 23 January 2009

(C) Springer Science+Business Media B.V. 2009

\begin{abstract}
In this written version of a pre-dinner-speech at the workshop "The Nature of Gravity" at ISSI I illustrate Pauli's science primarily with material that has not formally been published by him, but was communicated in detailed letters to eminent colleagues and friends.
\end{abstract}

Keywords Relativity $\cdot$ Quantum theory $\cdot$ Gauge theory $\cdot$ Kaluza-Klein theory

\section{Introduction}

Wolfgang Pauli was one of the most influential figures in twentieth-century science. In the foreword of the memorial volume to Pauli, edited by Markus Fierz and Victor Weisskopf (Fierz and Weisskopf 1960) — two former assistants of Pauli-Niels Bohr wrote about Pauli: "At the same time as the anecdotes around his personality grew into a veritable legend, he more and more became the very conscience of the community of theoretical physicists." There are few fields of physics on which Pauli's ideas have not left a significant imprint. From Pauli's enormous correspondence, edited by Karl von Meyenn (von Meyenn 1979-2005), and his studies in historical, epistemological and psychological questions, it becomes obvious that his searching mind embraced all aspects of human endeavor.

I knew Pauli only as a student. Beside attending his main courses, I saw him in action in the joint Theoretical Physics Seminar of ETH and the University, and in our general Physics Colloquium. Although it was a bit too early for me, I also visited some specialized lectures. In addition, I vividly remember a few public talks, like the famous one "On the earlier and more recent History of the Neutrino" that was given by Pauli immediately after the discovery of parity violation (Pauli 1994). Therefore, I can only talk about Pauli's science. Pauli was obviously a difficult and complex personality. Markus Fierz, who knew and understood him particularly well, once said in a talk: "Whoever knew him also felt that in this man the opposites of heavenly light and archaic darkness were having a tremendous impact."

N. Straumann $(\varangle)$

Institute for Theoretical Physics, University of Zurich, Zurich, Switzerland

e-mail: norbert.straumann@gmail.com 
And in a letter Markus Fierz wrote to me that a true biography would have to be written by a physicist with poetic gifts. In certain circles there is now a lot of interest in Pauli's special relationship with the psychiatrist Carl Gustav Jung. Only few physicist colleagues knew about this and corresponded with him on Jungian ideas and psychology in general. Pauli attached great importance to the analysis of his dreams and wrote them down in great number. Hundreds of pages of Pauli's notes are not yet published. There are scholars who are convinced that Pauli's thoughts about psychology are important. Others regard all this as mystical mumbo-jumbo. At any rate, Jung successfully helped Pauli to overcome his life crisis after his first marriage had broken up in 1930.

It would be hopeless and pointless to give an overview of Pauli's most important scientific contributions, especially since Charles Enz, the last of a prestigious chain of Pauli assistants, has published a very complete scientific biography (Enz 2002). Much of what Pauli has achieved has become an integral part of physics. ${ }^{1}$ You are all aware that he was one of the founders of quantum electrodynamics and quantum field theory in general, and he is, of course, the father of the neutrino. After some biographical remarks, and a sketch of his early work, I will select some important material that appeared only in letters. ${ }^{2}$

\section{A Brief Biography}

Let me begin with a few biographical remarks. Pauli was born in 1900, the year of Planck's great discovery. During the high school years Wolfgang developed into an infant prodigy familiar with the mathematics and physics of his day.

Pauli's scientific career started when he went to Munich in autumn 1918 to study theoretical physics with Arnold Sommerfeld, who had created a "nursery of theoretical physics". Just before he left Vienna on 22 September he had submitted his first published paper, devoted to the energy components of the gravitational field in general relativity (Pauli 1919a). As a 19-year-old student he then wrote two papers (Pauli 1919b, 1919c) about the recent brilliant unification attempt of Hermann Weyl (which can be considered in many ways as the origin of modern gauge theories). In one of them he computed the perihelion motion of Mercury and the light deflection for a field action which was then preferred by Weyl. From these first papers it becomes obvious that Pauli mastered the new field completely. Hermann Weyl was astonished. Already on 10 May, 1919, he wrote to Pauli from Zürich: "I am extremely pleased to be able to welcome you as a collaborator. However, it is almost inconceivable to me how you could possibly have succeeded at so young an age to get hold of all the means of knowledge and to acquire the liberty of thought that is needed to assimilate the theory of relativity."

Sommerfeld immediately recognized the extraordinary talent of Pauli and asked him to write a chapter on relativity in Encyklopädie der mathematischen Wissenschaften. Pauli was in his third term when he began to write this article. Within less than one year he finished this demanding job, beside his other studies at the university. With this article (Pauli

\footnotetext{
${ }^{1}$ For a review, see Straumann (2000).

${ }^{2}$ Pauli's Scientific Correspondence, admirably edited by Karl von Meyenn, is a source of wonderful insights. (Hopefully, the letters written in German will one day be translated into English.) In an obituary for Pauli his former assistant Rudolf Peierls wrote in 1960: "It would be impossible to list all the ideas, constructive or critical, by which he influenced the work of pupils and colleagues in innumerable letters. (...) In these letters, as well as in conversations, he would often discuss conjectures and intuitive judgements, which went far beyond anything he would regard as worthy of publication."
} 
1921, 1981) of 237 pages and almost 400 digested references Pauli established himself as a scientist of rare depth and surpassing synthetic and critical abilities. Einstein's reaction was very positive: "One wonders what to admire most, the psychological understanding for the development of ideas, the sureness of mathematical deduction, the profound physical insight, the capacity for lucid, systematic presentation, the knowledge of the literature, the complete treatment of the subject matter or the sureness of critical appraisal."

Pauli studied at the University of Munich for six semesters. At the time when his Encyclopedia article appeared, he obtained his doctorate with a dissertation on the hydrogen molecule ion $\mathrm{H}_{2}^{+}$in the old Bohr-Sommerfeld theory. In it the limitations of the old quantum theory showed up. About the faculties of the young Pauli Lise Meitner wrote to Pauli's widow Franca on 22 June 1959: "I often thought of and also have told it that in the fall of 1921 I have met Sommerfeld in Lund, and that he told me he had such a gifted student that the latter could not learn anything any more from him, but because of the university laws valid in Germany he had to sit through (absitzen) 6 semesters in order to make his doctorate. Therefore he, Sommerfeld had set his student on an encyclopedia article (...)." (From Enz 2002, p. 25.)

In the winter semester of 1921/22 Pauli was Max Born's assistant in Göttingen. During this time the two collaborated on the systematic application of astronomical perturbation theory to atomic physics. Already on 29 November, 1921, Born wrote to Einstein: "Little Pauli is very stimulating: I will never have again such a good assistant.” Well, Pauli's successor was Werner Heisenberg.

\section{Discovery of the Exclusion Principle}

Pauli's next stages were in Hamburg and Copenhagen. His work during these crucial years culminated with the proposal of his exclusion principle in December 1924. This was Pauli's most important contribution to physics, for which he received a belated Nobel Prize in 1945.

The discovery story begins in fall 1922 in Copenhagen when Pauli began to concentrate his efforts on the problem of the anomalous Zeeman effect. He later recalled: 'A colleague who met me strolling rather aimlessly in the beautiful streets of Copenhagen said to me in a friendly manner, "You look very unhappy"; whereupon I answered fiercely, "How can one look happy when he is thinking about the anomalous Zeeman effect?"' .

In a Princeton address in 1946 (Pauli 1946), Pauli tells us how he felt about the anomalous Zeeman effect in his early days:

The anomalous type of splitting was on the one hand especially fruitful because it exhibited beautiful and simple laws, but on the other hand it was hardly understandable, since very general assumptions concerning the electron, using classical theory as well as quantum theory, always led to a simple triplet. A closer investigation of this problem left me with the feeling that it was even more unapproachable (...). I could not find a satisfactory solution at that time, but succeeded, however, in generalizing Landé's analysis for the simpler case (in many respects) of very strong magnetic fields. This early work was of decisive importance for the finding of the exclusion principle.

This is not the place to even only sketch how Pauli arrived at his exclusion principle. ${ }^{3}$ At the time-before the advent of the new quantum mechanics - it was not at all on the horizon,

\footnotetext{
${ }^{3}$ For a detailed description, see, e.g., Straumann (2007), and references therein.
} 
because of two basic difficulties: (1) There were no general rules to translate a classical mechanical model into a coherent quantum theory, and (2) the spin degree of freedom was unknown. It is very impressive indeed how Pauli arrived at his principle on the basis of the fragile Bohr-Sommerfeld theory and the known spectroscopic material.

Initially Pauli was not sure to what extent his exclusion principle would hold good. In a letter to Bohr of 12 December 1924 Pauli writes "The conception, from which I start, is certainly nonsense. (...) However, I believe that what I am doing here is no greater nonsense than the hitherto existing interpretation of the complex structure. My nonsense is conjugate to the hitherto customary one." The exclusion principle was not immediately accepted, although it explained many facts of atomic physics. A few days after the letter to Bohr, Heisenberg wrote to Pauli on a postcard: "Today I have read your new work, and it is certain that I am the one who rejoices most about it, not only because you push the swindle to an unimagined, giddy height (by introducing individual electrons with 4 degrees of freedom) and thereby have broken all hitherto existing records of which you have insulted me. (...)."

At the end of his final paper (Pauli 1925) on the way to the exclusion principle, Pauli expresses the hope that a deeper understanding of quantum mechanics might enable us to derive the exclusion principle from more fundamental hypothesis. To some extent this hope was fulfilled in the framework of quantum field theory. Pauli's much later paper from 1940 (Pauli 1940) on the spin-statistics connection ends with:

In conclusion we wish to state, that according to our opinion the connection between spin and statistics is one of the most important applications of the special theory of relativity.

For the letters of Pauli on the exclusion principle, and the reactions of his influential colleagues, I refer to Vol. I of the Pauli Correspondence, edited by Karl von Meyenn (von Meyenn 1979-2005). Some passages are translated into English in the scientific biography by Charles Enz (2002).

\subsection{Some Side Remarks}

Let me end this brief account with some remarks about Pauli's fruitful Hamburg time. I begin with recollections of Otto Stern from a recorded interview with Res Jost-one of my most important teachers and a later close colleague-in Zurich that took place on December 2, 1961. During his Hamburg time, Pauli collaborated closely with Stern, who in 1922 had become professor for physical chemistry at the University of Hamburg. Stern said about Pauli: "But, of course, it was very nice with Pauli for, although he was thus highly learned, one could all the same really discuss physics with him. And ... you know, he was not allowed to enter our laboratory, because of the Pauli effect. Don't you know the famous Pauli effect? Jost: I know it all right, but I didn't know that this led to such consequences. Stern: Yet, now, as I said, we always went eating together, he always fetched me. But he did not enter, instead he only knocked, and I then came to the door and said I'm coming. Oh yes, we were very superstitious at the time. Jost: Did something ever happen? Stern: Alas, many things did happen. The number of Pauli effects, the guaranteed (verbürgten) Pauli effects, is enormously large." (Translation from Enz 2002, p. 149.)

In his obituary for Stern Rabi wrote: "Some of Pauli's great theoretical contributions came from Stern's suggestions, or rather questions; for example, the theory of magnetism of free electrons in metals." From Charly Enz and Armin Thellung-Pauli's last two assistants-I have learned that Pauli has also discussed the question of zero point energies extensively with Stern during his Hamburg time, before the advent of the new quantum 
mechanics. The following remarks may be of some interest since they are related to things discussed at our conference here.

As background I recall that Planck had introduced the zero-point energy with somewhat strange arguments in 1911. The physical role of the zero-point energy was much discussed in the early years of quantum theory. There was, for instance, a paper by Einstein and Stern in 1913 (Einstein 1987, Vol. 4, Doc. 11; see also the Editorial Note, p. 270 ff) that aroused widespread interest. In this two arguments in favor of the zero-point energy were given. The first had to do with the specific heat of rotating (diatomic) molecules. The authors developed an approximate theory of the energy of rotating molecules and came to the conclusion that the resulting specific heat agreed much better with recent experimental results by Arnold Eucken, if they included the zero-point energy. The second argument was based on a new derivation of Planck's radiation formula. In both arguments Einstein and Stern made a number of problematic assumptions, and in fall 1913 Einstein retracted their results. At the second Solvay Congress in late October 1913 Einstein said that he no longer believed in the zero-point energy, and in a letter to Ehrenfest (Einstein 1987, Vol. 5, Doc. 481) he wrote that the zero-point energy was "dead as a doornail".

In Hamburg Stern had calculated, but never published, the vapor pressure difference between the isotopes 20 and 22 of Neon (using Debye theory for the solid phase). He came to the conclusion that without zero-point energy this difference would be large enough for easy separation of the isotopes, which is not the case in reality. These considerations penetrated into Pauli's lectures on statistical mechanics (Pauli 1973) (which I attended). The theme was taken up in an article by Enz and Thellung (1960). This was originally written as a birthday gift for Pauli, but because of Pauli's early death, appeared in a memorial volume of Helv. Phys. Acta.

From Pauli's discussions with Enz and Thellung we know that Pauli estimated the influence of the zero-point energy of the radiation field - cut off at the classical electron radiuson the radius of the universe, and came to the conclusion that it "could not even reach to the moon".

When, as a student, I heard about this, I checked Pauli's unpublished ${ }^{4}$ remark by doing the following little calculation (which Pauli must have done):

In units with $\hbar=c=1$ the vacuum energy density of the radiation field is

$$
\langle\rho\rangle_{v a c}=\frac{8 \pi}{(2 \pi)^{3}} \int_{0}^{\omega_{\max }} \frac{\omega}{2} \omega^{2} d \omega=\frac{1}{8 \pi^{2}} \omega_{\max }^{4},
$$

with

$$
\omega_{\max }=\frac{2 \pi}{\lambda_{\max }}=\frac{2 \pi m_{e}}{\alpha} .
$$

The corresponding radius of the Einstein universe in Equation (2) would then be $\left(M_{p l} \equiv\right.$ $1 / \sqrt{G})$

$$
a=\frac{\alpha^{2}}{(2 \pi)^{\frac{2}{3}}} \frac{M_{p l}}{m_{e}} \frac{1}{m_{e}} \sim 31 \mathrm{~km} .
$$

This is indeed less than the distance to the moon. (It would be more consistent to use the curvature radius of the static de Sitter solution; the result is the same, up to the factor $\sqrt{3 / 2}$.)

\footnotetext{
${ }^{4}$ A trace of this is in Pauli's Handbuch article (Pauli 1933) on wave mechanics in the section where he discusses the meaning of the zero-point energy of the quantized radiation field. The last sentence of a brief paragraph ends with " $(. .$.$) and, as is evident from experience, neither does it produce any gravitational field".$
} 
Our present estimates of the vacuum energy, that possibly is responsible for an accelerated expansion of the universe, are not much better.

\subsection{Exclusion Principle and the New Quantum Mechanics}

On August 26, 1926, Dirac's paper containing the Fermi-Dirac distribution was communicated by R. Fowler to the Royal Society. This work was the basis of Fowler's theory of white $d w a r f s$. I find it remarkable that the quantum statistics of identical spin-1/2 particles found its first application in astrophysics. Pauli's exclusion principle was independently applied to statistical thermodynamics by Fermi. ${ }^{5}$ In the same year 1926, Pauli simplified Fermi's calculations, introducing the grand canonical ensemble into quantum statistics. As an application he studied the behavior of a gas in a magnetic field (paramagnetism).

Heisenberg and Dirac were the first who interpreted the exclusion principle in the context of Schrödinger's wave mechanics for systems of more than one particle. In these papers it was not yet clear how the spin had to be described in wave mechanics. (Heisenberg speaks of spin coordinates, but he does not say clearly what he means by this.) The definite formulation was soon provided by Pauli in a beautiful paper (Pauli 1927), in which he introduced his famous spin matrices and two-component spinor wave functions.

At this point the foundations of non-relativistic quantum mechanics had been completed in definite form. For a lively discussion of the role of the exclusion principle in physics and chemistry from this foundational period, I refer to Ehrenfest's opening laudation (Ehrenfest 1931) when Pauli received the Lorentz medal in 1931. This concluded with the words: "You must admit, Pauli, that if you would only partially repeal your prohibitions, you could relieve many of our practical worries, for example the traffic problem on our streets." According to Ehrenfest's assistant Casimir who was in the audience, Ehrenfest improvised something like this: "and you might also considerably reduce the expenditure for a beautiful, new, formal black suit" (quoted in Enz 2002, p. 258).

These remarks indicate the role of the exclusion principle for the stability of matter in bulk. A lot of insight and results on this central issue, both for ordinary matter (like stones) and self-gravitating bodies, have been obtained in more recent times, beginning with the work of Dyson and Lenard in 1967 (Dyson and Lenard 1967; Dyson and Lenard 1968). For further information, I highly recommend the review articles in Lieb's Selecta (Lieb 1991). (For a brief description, see Straumann 2007.)

\section{Pauli's Discovery of the Relation between Matrix Mechanics and Wave Mechanics (Letter to P. Jordan)}

On April 12, 1926 Pauli wrote a very remarkable letter to P. Jordan (von Meyenn 1979-2005, Vol. I, letter 131), just after the first communication of Schrödinger had appeared.

B.L. van der Waerden devoted his talk at the "Dirac conference" in Trieste in 1972 (van der Waerden 1973) almost entirely to this letter. In this Pauli established the connection between wave and matrix mechanics in a "logically irreproachable way, independent of Schrödinger. He never published the contents of this letter, but signed a carbon copy (which

\footnotetext{
${ }^{5}$ According to Max Born, Pascual Jordan was actually the first who discovered what came to be known as the Fermi-Dirac statistics. Unfortunately, Born, who was editor of the Zeitschrift für Physik, put Jordan's paper into his suitcase when he went for half a year to America in December of 1925, and forgot about it. For further details on this, I refer to the interesting article by E.L. Schucking (1999).
} 
is quite unusual) and he kept the letter in a plastic cover until his death" (van der Waerden's words).

I would like to go through this letter, which is also remarkable in other respects. At the same time it gives an impression of the enormous influence Pauli had through his extensive correspondence. Pauli's letters are an integral part of his work and thinking. It is also a wonderful experience to read at least some of them. The letter begins with

Dear Jordan,

Many thanks for your last letter and for looking through the proof sheets. Today I want to write neither about my Handbuch-Article nor about multiple quanta; I will rather tell you the results of some considerations of mine connected with Schrödinger's paper 'Quantisierung als Eigenwertproblem' which just appeared in the Annalen der Physik. I feel that this paper is to be counted among the most important recent publications. Please read it carefully and with devotion.

Of course I have at once asked myself how his results are connected with those of the Göttingen Mechanics. I think I have now completely clarified this connection. I have found that the energy values from Schrödinger's approach are always the same as those of the Göttingen Mechanics, and that from Schrödinger's functions $\psi$, which describe the eigenvibrations, one can in a quite simple and general way construct matrices satisfying the equations of the Göttingen Mechanics. Thus at the same time a rather deep connection between the Göttingen Mechanics and the Einstein-de Boglie Radiation Field is established.

To make this connection as clear as possible, I shall first expose Schrödinger's approach, styled a little differently.

Pauli does not start with Schrödinger's stationary equation of his 'First Communication', whose justification I find, by the way, rather obscure. ${ }^{6}$ Pauli first derived what we now call the Klein-Gordon equation. ${ }^{7} \mathrm{He}$ starts from the relativistically invariant Einstein-de Broglie relations $\mathbf{p}=\hbar \mathbf{k}, E=\hbar \omega$, and inserts these into the relativistic mechanical equation

$$
E-V=E_{k i n}=\sqrt{c^{2} \mathbf{p}^{2}+\left(m c^{2}\right)^{2}} \Rightarrow \mathbf{p}^{2}=\hbar^{2} \mathbf{k}^{2}=\frac{1}{c^{2}}\left[(E-V)^{2}-\left(m c^{2}\right)^{2}\right] .
$$

If this is inserted into the stationary wave equation

$$
\left(\triangle+k^{2}\right) \psi=0
$$

one obtains the stationary Klein-Gordon equation

$$
\left[\triangle+\left(\frac{E-V}{\hbar c}\right)^{2}-\left(\frac{m c}{\hbar}\right)^{2}\right] \psi=0 .
$$

(Actually, Pauli first arrives at a time-dependent equation, which is, however, different from the Klein-Gordon equation, except in the free case.) Then Pauli considers the non-relativistic limit and writes: "This equation is given in Schrödinger's paper, and he also shows how it can be derived from a Variational Principle". After a remark about the analogy with the difference between Geometrical Optics and Wave Optics, he says:

\footnotetext{
${ }^{6}$ A profound justification, based on the mechanical-optical analogy, was given in the Second Communication.

${ }^{7}$ From Schrödinger's research notes we know that he studied this equation before he had the Schrödinger equation, but abandoned it because it gave the wrong fine structure for hydrogen.
} 
"Next comes my own contribution, namely the connection with the Göttingen Mechanics." With the complete set of eigenfunctions of the stationary Schrödinger equation he associates to operators of the Schrödinger theory matrices, and verifies that these "satisfy the equations of the Göttingen Mechanics". Since we are all familiar with this, no further comments are necessary.

The following remark is noteworthy. Pauli treats the wave function without saying as a complex field, while Schrödinger maintained that " $\psi$ is surely fundamentally a real function ...". Only in the fourth communication he arrived at the conviction that it is actually complex, but states in the last of his major papers on wave mechanics "that a certain difficulty no doubt still lies in the use of a complex wave function." (For more on this, see Straumann 2001.)

During the short time after the First Communication of Schrödinger had appeared, Pauli did more:

I have calculated the oscillator and rotator according to Schrödinger. Further the HönlKronig-formulae for the intensity of Zeeman components are easy consequences of the properties of the spherical harmonics. Perturbation theory can be carried over completely into the new theory, and the same thing holds for the transformation to principal axes, which in general is necessary if degenerations (multiple eigenvalues) are cancelled by external fields of force. At the moment I am occupying myself with the calculation of transition probabilities in hydrogen from the eigenfunctions calculated by Schrödinger. For the Balmer lines finite rational expressions seem to come out. For the continuous spectrum the situation is more complicated: the exact mathematical formulation is not yet quite clear to me.

On this Pauli was again a bit too late to submit a paper. The one by Schrödinger was submitted on March 18, who wrote a month later to Sommerfeld: "With Pauli I have exchanged a few long letters. He is indeed a phenomenal fellow. How fast he has obtained everything! In a tenth of the time I have been using." [Mit Pauli habe ich ein paar lange Briefe gewechselt. Er ist schon ein phänomenaler Kerl. Wie der wieder alles schnell heraussen gehabt hat! In einem Zehntel der Zeit, die ich dazu gebraucht hab.]

The letter of Pauli to Jordan ends with: "Cordial greetings for you and the other people in Göttingen (especially to Born, in case he is back from America; please show him this letter)."

Supplementary Remarks. From a letter of Pauli to Schrödinger late in 1926 it is clear that he independently discovered the gauge invariance. In this letter Pauli begins by saying that at first sight the relativistic wave equation does not only contain the field strengths, but also the absolute values of the 4-potential. However, he adds: "Thanks God this is only apparent", and he gives the formulae for what we call gauge invariance of the relativistic Kein-Gordon equation. Again, Pauli did not publish the content of this letter, because he learned from Schrödinger's answer about a paper Schrödinger had just submitted to the 'Annalen' (two days before Pauli had written his letter). However, Schrödinger says in his paper nothing about gauge invariance.

\section{On Pauli's Invention of non-Abelian Kaluza-Klein Theory in 1953}

There are documents which show that Wolfgang Pauli constructed in 1953 the first consistent generalization of the five-dimensional theory of Kaluza, Klein, Fock and others to a higher 
dimensional internal space. Because he saw no way to give masses to the gauge bosons, he refrained from publishing his results formally. This is still a largely unknown chapter of the early history of non-Abelian gauge and Kaluza-Klein theories.

Pauli described his detailed attempt of a non-Abelian generalization of Kaluza-Klein theories extensively in some letters to A. Pais, which have been published in Vol. IV, Part II of Pauli's collected letters (Pauli 1999), and also in two seminars in Zürich on November 16 and 23, 1953. The latter have later been written up in Italian by Pauli's pupil P. Gulmanelli (Gulmanelli 1954). An English translation of these notes by P. Minkowski is now available on his home page. By specialization (independence of spinor fields on internal space) Pauli got all important formulae of Yang and Mills, as he later (Feb. 1954) pointed out in a letter to Yang (Pauli 1954), after a talk of Yang in Princeton. Pauli did not publish his study, because he was convinced that "one will always obtain vector mesons with rest mass zero" (Pauli to Pais, 6 Dec., 1953).

\subsection{The Pauli Letters to Pais}

At the Lorentz-Kammerlingh Onnes conference in Leiden (22-27 June 1953) A. Pais talked about an attempt of describing nuclear forces based on isospin symmetry and baryon number conservation. In this contribution he introduced fields, which do not only depend on the spacetime coordinates $x$, but also on the coordinates $\omega$ of an internal isospin space. The isospin group acted, however, globally, i.e., in a spacetime-independent manner.

During the discussion following the talk by Pais, Pauli said:

I would like to ask in this connection whether the transformation group with constant phases can be amplified in a way analogous to the gauge group for electromagnetic potentials in such a way that the meson-nucleon interaction is connected with the amplified group (...).

Stimulated by this discussion, Pauli worked on the problem, and wrote on July 25, 1953 a long technical letter to Pais (Pauli 1953a), with the motto: "Ad usum Delfini only". This letter begins with a personal part in which Pauli says that "the whole note for you is of course written in order to drive you further into the real virgin-country". The note has the interesting title:

Written down July 22-25 1953, in order to see how it looks. Meson-Nucleon Interaction and Differential Geometry.

In this manuscript, Pauli generalizes the original Kaluza-Klein theory to a six-dimensional space and arrives through dimensional reduction at the essentials of an $S U$ (2) gauge theory. The extra-dimensions form a two-sphere $S^{2}$ with space-time dependent metrics on which the $S U(2)$ operates in a space-time-dependent manner. Pauli emphasizes that this transformation group "seems to me therefore the natural generalization of the gauge-group in case of a two-dimensional spherical surface". He then develops in 'local language' the geometry of what we now call a fibre bundle with a homogeneous space as typical fiber (in this case $S U(2) / U(1)$ ). Since it is somewhat difficult to understand exactly what Pauli did, we give some details, using more familiar formulations and notations (O'Raifeartaigh and Straumann 2000).

Pauli considers the six-dimensional total space $M \times S^{2}$, where $S^{2}$ is the two-sphere on which $S O(3)$ acts in the canonical manner. He distinguishes among the diffeomorphisms (coordinate transformations) those which leave the space-time manifold $M$ pointwise fixed and induce space-time-dependent rotations on $S^{2}$ :

$$
(x, y) \rightarrow(x, R(x) \cdot y) .
$$


Then Pauli postulates a metric on $M \times S^{2}$ that is supposed to satisfy three assumptions. These led him to what is now called the non-Abelian Kaluza-Klein ansatz: The metric $\hat{g}$ on the total space is constructed from a space-time metric $g$, the standard metric $\gamma$ on $S^{2}$, and a Lie-algebra-valued 1-form,

$$
A=A^{a} T_{a}, \quad A^{a}=A_{\mu}^{a} d x^{\mu},
$$

on $M\left(T_{a}, a=1,2,3\right.$, are the standard generators of the Lie algebra of $\left.S O(3)\right)$ as follows: If $K_{a}^{i} \partial / \partial y^{i}$ are the three Killing fields on $S^{2}$, then

$$
\hat{g}=g-\gamma_{i j}\left[d y^{i}+K_{a}^{i}(y) A^{a}\right] \otimes\left[d y^{j}+K_{a}^{j}(y) A^{a}\right] .
$$

In particular, the non-diagonal metric components are

$$
\hat{g}_{\mu i}=A_{\mu}^{a}(x) \gamma_{i j} K_{a}^{j} .
$$

Pauli does not say that the coefficients of $A_{\mu}^{a}$ in (4) are the components of the three independent Killing fields. This is, however, his result, which he formulates in terms of homogeneous coordinates for $S^{2}$. He determines the transformation behavior of $A_{\mu}^{a}$ under the group (1) and finds in matrix notation what he calls "the generalization of the gauge group":

$$
A_{\mu} \rightarrow R^{-1} A_{\mu} R+R^{-1} \partial_{\mu} R
$$

With the help of $A_{\mu}$, he defines a covariant derivative, which is used to derive "field strengths" by applying a generalized curl to $A_{\mu}$. This is exactly the field strength that was later introduced by Yang and Mills. To our knowledge, apart from Klein's 1938 paper, it appears here for the first time. Pauli says that "this is the true physical field, the analog of the field strength" and he formulates what he considers to be his "main result":

The vanishing of the field strength is necessary and sufficient for the $A_{\mu}^{a}(x)$ in the whole space to be transformable to zero.

It is somewhat astonishing that Pauli did not work out the Ricci scalar for $\hat{g}$ as for the Kaluza-Klein theory. One reason may be connected with his remark on the Kaluza-Klein theory in Note 23 of his relativity article (Pauli 1981) concerning the five-dimensional curvature scalar (p. 230):

There is, however, no justification for the particular choice of the five-dimensional curvature scalar $P$ as integrand of the action integral, from the standpoint of the restricted group of the cylindrical metric (gauge group). The open problem of finding such a justification seems to point to an amplification of the transformation group.

In a second letter (Pauli 1953b), Pauli also studies the dimensionally reduced Dirac equation and arrives at a mass operator that is closely related to the Dirac operator in internal space $\left(S^{2}, \gamma\right)$. The eigenvalues of the latter operator had been determined by him long before (Pauli 1939). Pauli concludes with the statement: "So this leads to some rather unphysical shadow particles."

Pauli's main concern was that the gauge bosons had to be massless, as in quantum electrodynamics. He emphasized this mass problem repeatedly, most explicitly in the second letter (Pauli 1953b) to Pais on December 6, 1953, after he had made some new calculations and had given the two seminar lectures in Zurich already mentioned. He adds to the Lagrangian what we now call the Yang-Mills term for the field strengths and says that "one 
will always obtain vector mesons with rest-mass zero (and the rest-mass if at all finite, will always remain zero by all interactions with nucleons permitting the gauge group)." To this Pauli adds: "One could try to find other meson fields", and he mentions, in particular, the scalar fields which appear in the dimensional reduction of the higher-dimensional metric. In view of the Higgs mechanism this is an interesting remark.

Pauli learned about the related work of Yang and Mills in late February, 1954, during a stay in Princeton, when Yang was invited by Oppenheimer to return to Princeton and give a seminar on his joint work with Mills. About this seminar Yang reports (Yang 1983): "Soon after my seminar began, when I had written down on the blackboard $\left(\partial_{\mu}-i \epsilon B_{\mu}\right) \Psi$, Pauli asked: What is the mass of this field $B_{\mu}$ ?, I said we did not know. Then I resumed my presentation, but soon Pauli asked the same question again. I said something to the effect that was a very complicated problem, we had worked on it and had come to no conclusion. I still remember his repartee: 'That is no sufficient excuse.' I was so taken aback that I decided, after a few moments' hesitation to sit down. There was general embarrassment. Finally Oppenheimer said, 'we should let Frank proceed.' Then I resumed and Pauli did not ask any more questions during the seminar." (For more on this encounter, see Yang 1983.)

In a letter to Yang (Pauli 1954) shortly after Yang's Princeton seminar, Pauli repeats: "But I was and still am disgusted and discouraged of the vector field corresponding to particles with zero rest-mass (I do not take your excuses for it with 'complications' seriously) and the difficulty with the group due to the distinction of the electromagnetic field remains." Formally, Pauli had, however, all important equations, as he shows in detail, and he concludes the letter with the sentence: "On the other hand you see, that your equations can easily be generalized to include the $\omega$-space" (the internal space). As already mentioned, the technical details have been written up by Pauli's pupil P. Gulmanelli (Gulmanelli 1954) and have recently been translated by P. Minkowski from Italian to English.

I hope that my scattered remarks have at least indicated that Wolfgang Pauli was a great man of uncompromising scientific honesty, to whom his own words (Pauli 1984) on Einstein apply equally well: "His life anticipating the future will forever remind us of the ideal—under threat in our time—of spiritual, contemplative man, his thoughts calmly and unswervingly bent on the great problems of the structure of the cosmos."

Acknowledgements I wish to thank the ISSI Institute and the local organizers, Martin Huber and Rudolf Treumann, for the opportunity to attend such an interesting workshop. I also thank Engelbert Schücking for comments.

\section{References}

F.J. Dyson, A. Lenard, Stability of matter, I and II. J. Math. Phys. 8, 423-434 (1967)

F.J. Dyson, A. Lenard, Stability of matter, I and II. J. Math. Phys. 9, 698-711 (1968)

P. Ehrenfest, Ansprache zur Verleihung der Lorentzmedaille an Professor Wolfgang Pauli am 31. Oktober 1931, Versl. Akad. Amsterdam 40, 121-126 (1931)

A. Einstein, The Collected Papers of Albert Einstein, Vols. 1-10 (Princeton University Press, 1987). See also: http://www.einstein.caltech.edu/

Ch.P. Enz, No Time to Be Brief: A Scientific Biography of Wolfgang Pauli (Oxford University Press, New York, 2002)

C.P. Enz, A. Thellung, Helv. Phys. Acta 33, 839 (1960)

M. Fierz, V. Weisskopf (eds.), Theoretical Physics in the Twentieth Century: A Memorial Volume to Wolfgang Pauli (Interscience, New York, 1960)

P. Gulmanelli, Su una Teoria dello Spin Isotropico, Pubblicazioni della Sezione di Milano dell'istituto Nazionale di Fisica Nucleare (Casa Editrice Pleion, Milano, 1954)

E.H. Lieb, The Stability of Matter: From Atoms to Stars, Selecta of Elliott H. Lieb (Springer, Berlin, 1991)

L. O’Raifeartaigh, N. Straumann, Rev. Mod. Phys. 72, 1-23 (2000) 
W. Pauli Jr, Physikalische Zeitschrift 20, 25 (1919a)

W. Pauli Jr, Physikalische Zeitschrift 20, 457 (1919b)

W. Pauli Jr, Verhandlungen der Deutschen Physikalischen Gesellschaft 21, 742 (1919c)

W. Pauli, in Encyklopädie der mathematischen Wissenschaften, vol. 19 (Teubner, Leipzig, 1921), pp. 539-775 new German Edition: Edited and annotated by D. Giulini, including Pauli's own supplementary notes from 1956 (Springer, Berlin, 2000)

W. Pauli, Über den Zusammenhang des Abschlusses der Elektronengruppen im Atom mit der Komplexstruktur der Spektren. Z. Phys. 31, 765-783 (1925)

W. Pauli, Zur Quantenmechanik des magnetischen Elektrons. Z. Physik 43, 601-623 (1927)

W. Pauli, Die allgemeinen Prinzipien der Wellenmechanik, Handbuch der Physik, vol. XXIV (1933). New edition by N. Straumann (Springer, 1990); see Appendix III, p. 202

W. Pauli, Helv. Phys. Acta 12, 147 (1939)

W. Pauli, On the connection between spin and statistics. Phys. Rev. 58, 716 (1940)

W. Pauli, Remarks on the history of the exclusion principle. Science 103, 213-215 (1946)

Pauli to Pais, Letter [1614] in (Pauli 1999)

Pauli to Pais, Letter [1682] in (Pauli 1999)

Pauli to Yang, Letter [1727] in (Pauli 1999)

W. Pauli, in Pauli Lectures on Physics, ed. by C.P. Enz, vol. 4 (MIT, Cambridge, 1973) especially Sect. 20

W. Pauli, Theory of Relativity (Dover, New York, 1981)

W. Pauli, Translation from the article Impressionen über Albert Einstein, in Aufsätze und Vorträge über Physik und Erkenntnistheorie (Vieweg, Braunschweig, 1961), p. 81. Reprinted as Physik und Erkenntnistheorie (Vieweg, Braunschweig, 1984)

W. Pauli, in Writings on Physics and Philosophy, ed. by Ch.P. Enz, K. von Meyenn (Springer, Berlin, 1994), translated by R. Schlapp

W. Pauli, in (Meyenn 1979-2005), Vol. IV, Part II

E.L. Schucking, Jordan, Pauli, Politics, Brecht, and a variable gravitational constant, Physics Today, October 1999, pp. 26-31

N. Straumann, On Wolfgang Pauli's most important contributions to physics. Opening talk at the symposium: Wolfgang Pauli and Modern Physics, in honor of the 100th anniversary of Wolfgang Pauli's birthday, ETH (Zurich), May 4-6 2000. arXiv:physics/0010003

N. Straumann, Schrödinger's Entdeckung der Wellenmechanik. arXiv:quant-ph/0110097

N. Straumann, The role of the exclusion principle for atoms to stars: a historical account. Int. Rev. Phys. 1, 184-196 (2007). arXiv:quant-ph/0403199

B.L. van der Waerden, in The Physicist's Conception of Nature, ed. by J. Mehra (Reidel, Dortrecht, 1973) p. 315

K. von Meyenn (ed.), Wolfgang Pauli: Scientific Correspondence with Bohr, Einstein, Heisenberg, a.O., Vol. I-IV, volume 2,6,11,14,15,17,18 of Sources in the History of Mathematics and Physical Sciences (Springer, Heidelberg and New York, 1979-2005)

C.N. Yang, Selected papers 1945-1980 with Commentary (Freeman, San Francisco, 1983), p. 525 